\title{
Serum N-terminal pro-B-type natriuretic peptide levels are associated with left atrial dilation, resting left ventricular outflow tract gradient, and pulmonary hypertension in patients with hypertrophic cardiomyopathy
}

\author{
Adam Gębka ${ }^{1}$, Renata Rajtar-Salwa ${ }^{1}$, Artur Dziewierz², Paweł Petkow-Dimitrow ${ }^{2}$ \\ ${ }^{1}$ Second Department of Cardiology and Cardiovascular Interventions, University Hospital, Krakow, Poland \\ ${ }^{2}$ Second Department of Cardiology, Jagiellonian University Medical College, Krakow, Poland
}

Adv Interv Cardiol 2020; 16, 1 (59): 97-101

DOI: https://doi.org/10.5114/aic.2019.91516

\begin{abstract}
A bstract
Introduction: N-terminal pro-B-type natriuretic peptide (NT-proBNP) can be a marker of left ventricle (LV) pressure overload in hypertrophic cardiomyopathy (HCM). The different clinical characteristics of HCM might correspond to the degree of NT-proBNP increase.

Aim: This study aimed to establish whether the left atrium (LA) dimension, left ventricle outflow tract (LVOT) gradient, and pulmonary hypertension influence NT-proBNP serum levels in patients with HCM.

Material and methods: In $62 \mathrm{HCM}$ patients (32 males and 30 females, mean age $31 \pm 11$ years), echocardiography with LV outflow tract gradient provocation was performed using natural stimuli > $30 \mathrm{~mm} \mathrm{Hg}$ (NOHCM - 36 patients, POHCM - 12 patients, HOCM - 14 patients).

Results: Smaller LAD was associated with a lower NT-proBNP/ULN level $(p=0.001)$. In contrast, smaller vs. larger LAD subgroups did not differ in NT-proBNP level $(p=0.42)$. Both NT-proBNP/ULN and NTproBNP were significantly elevated in the subgroup with lager LAA. The absolute value of NT-proBNP was significantly higher in the HOCM subgroup (NOHCM vs. POHCM vs. HOCM ( $p=$ 0.02). Similarly, NT-proBNP/ULN was significantly higher in the HOCM subgroup (NOHCM vs. POHCM vs. HOCM, $p=0.00047$ ). This elevated value of biomarker is related to pulmonary hypertension.

Conclusions: Increased NT-proBNP/ULN is positively associated with larger LAD and LAA, while elevated NTproBNP is only associated with larger LAA. The highest levels of both NT-proBNP and NTproBNP/ULN were associated with HOCM and pulmonary hypertension, whereas biomarker levels were comparably lower in both the POHCM and NOHCM.
\end{abstract}

Key words: hypertrophic cardiomyopathy, biomarker, NT-proBNP, pulmonary hypertension.

Su m m a ry

Increases in N-terminal pro-B-type natriuretic peptide (NT-proBNP)/upper limit of normal (ULN) are related to larger left atrial diameter (LAD) and LA area (LAA), while more elevated NT-proBNP is associated only with larger LAA. The highest levels of both NT-proBNP and NT-proBNP/ULN were associated with obstructive hypertrophic cardiomyopathy (HOCM), whereas biomarker levels were comparably lower in provocable obstructive HCM (PHOCM) and non-obstructive HCM (NOHCM).

\section{Introduction}

In hypertrophic cardiomyopathy (HCM), left atrial (LA) enlargement might be associated with cardiovascular death via the following mechanisms: as a risk factor for sudden car- diac death $(S C D)$ due to ventricular fibrillation or as a cause of cardioembolic stroke due to atrial fibrillation (AF) [1, 2].

In the 2014 European Society of Cardiology (ESC) guidelines [1], LA diameter (LAD) is measured in the

Corresponding author:

Prof. Paweł Petkow-Dimitrow MD, PhD, Second Department of Cardiology, Jagiellonian University Medical College, Krakow,

phone: +48 12 4247170, e-mail: dimitrow@mp.pl

Received: 30.07.2019, accepted: 25.11.2019. 
parasternal long-axis view as the anteroposterior linear diameter at the end-systolic phase. LA enlargement is proposed as a predictor of $A F$ and stroke in patients with HCM. Increased risk of AF is suggested as a cut-off value for LAD of $\geq 4.5 \mathrm{~cm}$. Recently, it has been postulated that when using the planimetric method, in which the $L A$ area (LAA) is measured from the apical view, a cut-off value of $\geq 28 \mathrm{~cm}^{2}$ for LAA is a strong predictor of $A F$ in patients with HCM [3]. Considering these two thresholds, the first aim of our study was to compare NT-proBNP and (standardised to age and gender) NT-pro-BNP/upper limit of normal (ULN) levels between patients with smaller and larger LA (as measured with both the above methods (LAD, LAA)) in patients without AF.

Additionally, we assessed the role of elevated NT-proBNP and NT-proBNP/UNL as a maker of provocable left ventricular outflow tract (LVOT) gradient $\geq 30 \mathrm{~mm} \mathrm{Hg}$ by comparing these values (absolute and standardised) between three subgroups of patients: with non-obstructive $\mathrm{HCM}$ (NOHCM), provocable obstructive HCM (PHOCM), and resting obstructive HCM (HOCM) at multiple measurements (stable obstruction at every echocardiography examination). Finally, we compared the biomarker level between subgroups of HCM patients (with and without pulmonary hypertension) and calculated the correlation between the biomarker and right ventricular dimension measured in the four-chamber apical view.

In the HCM subgroup, biomarkers are helpful for disease assessment $[4,5]$. Troponin is an independent predictor useful for identifying myocardial fibrosis, while plasma NT-proBNP is only associated with myocardial fibrosis on univariate analysis [5]. Combined measurements of serum troponin with maximal wall thickness further improve its power for detecting myocardial fibrosis in patients with HOCM [5].

\section{Aim}

This study aimed to establish whether the LA dimension, LVOT gradient, and pulmonary hypertension influence NT-proBNP serum levels in patients with HCM.

\section{Material and methods}

A total of 62 consecutive patients with HCM (mean age: $31 \pm 11$ years; 32 men and 30 women) were recruited to the study. Sinus rhythm was present in 51 patients. Informed consent was obtained from each participant. All patients met standard diagnostic criteria for HCM [1]. The exclusion criteria were as follows: ST-segment or non-ST-segment elevation myocardial infarction (current or previous), history of alcohol septal ablation, dilated LV cavity, decreased LV contractibility (end-stage, burn-offstage of HCM), or elevated creatinine level.

Patients on current pharmacotherapy were examined by echocardiography with LVOT gradient provocation by natural stimuli (orthostatic test and Valsalva test $[1,6,7]$ ) and were classified into the subgroups with NOHCM, PHOCM, and HOCM. Pulmonary hypertension probability was calculated by echocardiography according to available guidelines [8]. Additionally, we studied the relationship between pulmonary hypertension probability $(\mathrm{PH})$ and NT-proBNP, as well as NT-proBNP/ULN.

After the measurement, we defined 19 patients as having a high probability of pulmonary hypertension $(\mathrm{PH})$ and 43 as having a low probability. NT-proBNP and NT-proBNP/ULN were correlated with the RV dimension measured in the four-chamber apical view.

Roche Diagnostics NT-ProBNP electrochemiluminescence immunoassay was performed. The NT-proBNP levels were measured as absolute values and as a transformed value where the absolute value was standardised according to sex and age (based on the manufacturer's guidelines, http://www.rochecanada.com/content/dam/ roche_canada/en_CA/documents/package_inserts/ ProBNPII-04842464190-EN-V9-CAN.pdf). Values greater than the $97.5^{\text {th }}$ percentile for age and gender (upper limit of normal (ULN)) were considered abnormal. Therefore, results were expressed as the ratio of the NT-pro-BNP to age, sex-matched ULN, and the ratio value > 1.0 was abnormal [9]. This standardisation of NT-proBNP provides a normal distribution of data, whereas the absolute values were distributed abnormally. In this situation, we do not need to perform logarithmic transformation for artificial calculation.

\section{Statistical analysis}

Continuous variables were presented as mean (SD) or median (interquartile range, IQR). NT-proBNP levels (non-normal distribution) were compared by the Mann-Whitney test in the subgroup comparison (LAD smaller versus larger and LAA smaller versus larger, $\mathrm{PH}$ positive vs. $\mathrm{PH}$ negative). The NT-proBNP levels were compared between the three subgroups of patients with NOHCM, PHOCM, and HOCM using the Kruskal-Wallis test. Additional data analysis was performed with analysis of variance (one-way ANOVA) with post hoc Tuckey test.

The values of standardised NT-proBNP/ULN with a normal distribution (according to Kolmogorov-Smirnov test) were compared using Student's t-test for LAD, LAA, and $\mathrm{PH}$ analysis. The ANOVA test was used for NOHCM, PHOCM, and HOCM comparisons. A $p$-value of $<0.05$ was considered statistically significant.

\section{Results}

The baseline characteristics of patients with HCM are shown in Table I. Smaller LAD was associated with lower NT-proBNP/ULN level (3.91 \pm 3.69 vs. $7.09 \pm 7.41, p=$ $0.001)$. In contrast, smaller vs. larger LAD subgroups did not differ in NT-proBNP level (466.45 (180.8-894.25) vs. 551.7 (256.5-1279) pg/ml, $p=0.42$ ). Both NT-proBNP/ ULN and NT-proBNP were significantly higher in the sub- 
group with lager LAA (3.31 \pm 2.69 vs. $10.89 \pm 7.94, p=$ 0.000002 and 383 (187-778) vs. 1601 (551-2573) pg/ml, $p=0.002$ ).

The absolute value of NT-proBNP was significantly higher in the HOCM subgroup (NOHCM vs. PHOCM vs. HOCM, 539 (339-1035) vs. 567 (142-969) vs. 1909 (1019-3883) $\mathrm{pg} / \mathrm{ml} p=0.02$ ) (Figure 1).

Similarly, NT-proBNP/ULN was significantly higher in the HOCM subgroup (NOHCM vs. PHOCM vs. HOCM $4.31 \pm 0.81$ vs. $3.90 \pm 1.42$ vs. $10.69 \pm 1.41, p=0.00047$ ) (Figure 2).

Additional data analysis was performed with analysis of variance (one-way ANOVA) with post hoc Tuckey test. For NT-proBNP and NT-proBNP/ULN, the one-way ANOVA test was statistically significant $(p<0.001$ and $p=0.006)$ between all subgroups. Differences in the Tuckey HSD test were significant between the following subgroups: NOHCM vs. HOCM $p<0.001$ and POHCM vs. HOCM ( $p=$ 0.020). The mean values of the LVOT max gradient were statistically different $(p<0.001)$ both in the ANOVA and Tuckey HSD tests for all comparisons between subgroups.

Moreover, we studied the relationship between pulmonary hypertension and NT-proBNP (Figure 3), as well as NT-proBNP/ULN (Figure 4). We detected 19 patients with $\mathrm{PH}$ and 43 without $\mathrm{PH}$. Patients with $\mathrm{PH}$ vs. non$\mathrm{PH}$ had significantly higher levels of NT-proBNP (1664 (904.5-2679) vs. 367.3 (187.6-900), $p<0.000002$ ).

When using the standardised value of NT-proBNP/ ULN, patients with PH vs. non-PH (Figure 4) also had a significantly higher level of NT-proBNP/ULN (9.41 \pm 6.91 vs. 3.82, $p=0.00011$ ).

In contrast to the biomarker and $\mathrm{PH}$ relationships, both NT-proBNP and NT-proBNP/ULN did not correlate with the RV dimension measured in the four-chamber view $(r=0.01207, p=\mathrm{NS}$; and $r=0.0056, p=\mathrm{NS})$.

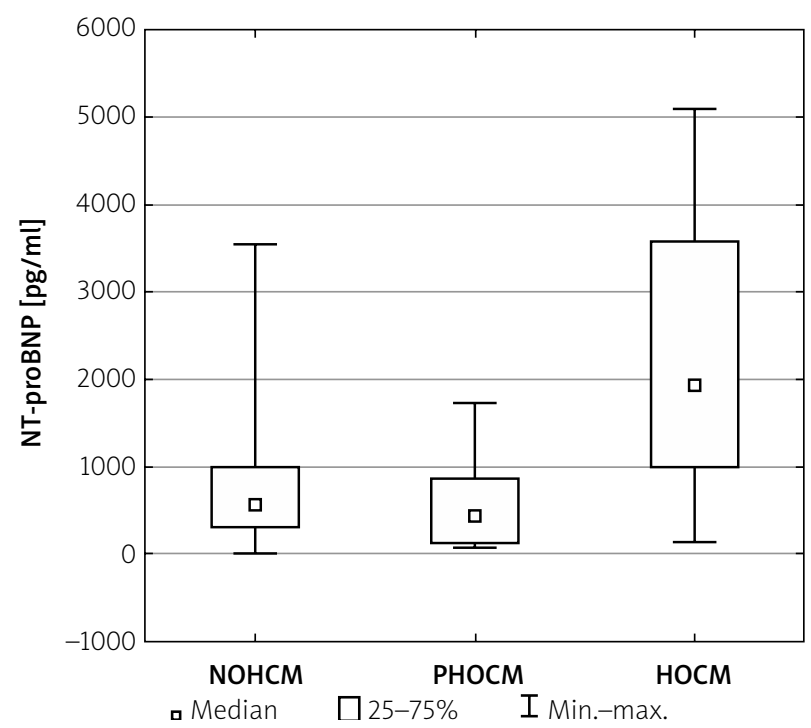

Figure 1. Comparison of NT-proBNP between NOHCM vs. PHOCM vs. HOCM subgroups
Table I. Baseline characteristics of the patients

\begin{tabular}{lc} 
Parameter & Value \\
\hline NYHA class & $2.2 \pm 0.7$ \\
\hline CCS class & $1.2 \pm 0.4$ \\
\hline Atrial fibrillation $(n)$ & 11 \\
\hline Syncope $(n)$ & 23 \\
\hline Sudden death in family history $(n)$ & 22 \\
\hline NSVT in Holter $(n)$ & 24 \\
\hline Maximum LV thickness [mm] & $22.6 \pm 4.9$ \\
\hline Resting LVOT gradient, $>30 \mathrm{~mm} \mathrm{Hg},(n)$ & 14 \\
\hline Provocable LVOT gradient, $\geq 30 \mathrm{~mm} \mathrm{Hg},(n)$ & 12 \\
\hline Left atrial diameter, mean $(\mathrm{SD})[\mathrm{mm}]$ & $4.89 \pm 0.81$ \\
\hline Pulmonary hypertension $(n)$ & 19
\end{tabular}

CCS - Canadian Cardiovascular Society, LVOT - left ventricular outflow tract, LV-left ventricular, NSVT-non-sustained ventricular tachycardia, NYHA - New York Heart Association.

\section{Discussion}

Here we report several novel findings, including biomarker verification of $L A D$ and $L A A$ as a risk factor for $A F$, and that an LAA $>28 \mathrm{~cm}^{2}$ is probably a better predictor than $L A D>4.5 \mathrm{~cm}$. Also, a new finding generated in our study is that highly and significantly elevated NT-proBNP and NT-proBNP/ULN levels are associated with resting LVOT gradient > $30 \mathrm{~mm} \mathrm{Hg}$ (stable LVOT obstruction). On the other hand, significantly lower NT-proBNP and NT-proBNP/ULN levels (but still elevated value) did not predict provocable (labile, latent) LVOT gradient $>30 \mathrm{~mm} \mathrm{Hg}$. This is because the biomarker levels were similar be-

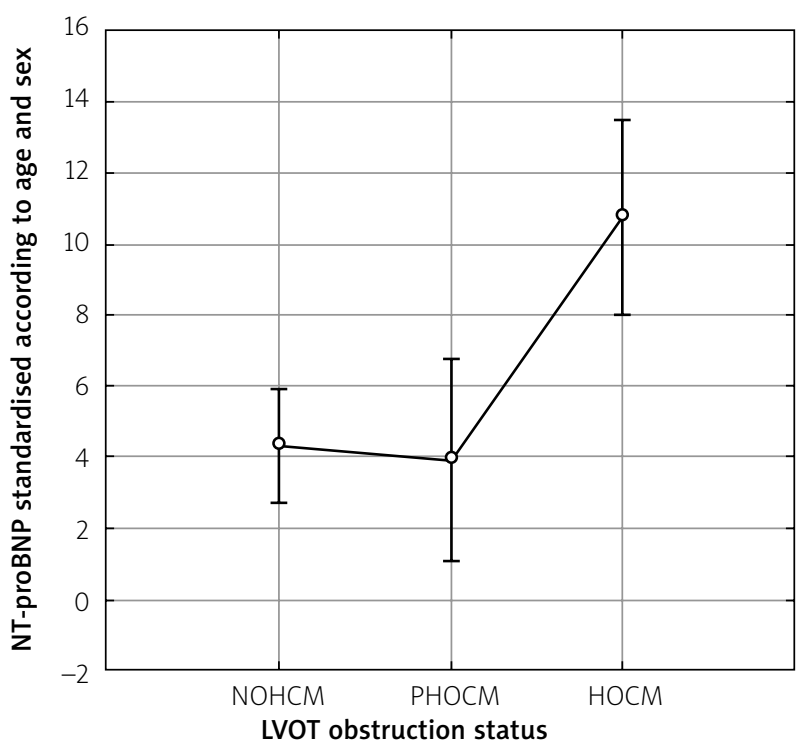

Figure 2. Comparison of NT-proBNP/ULN standardised according to age and sex between NOHCM vs. PHOCM vs. HOCM 


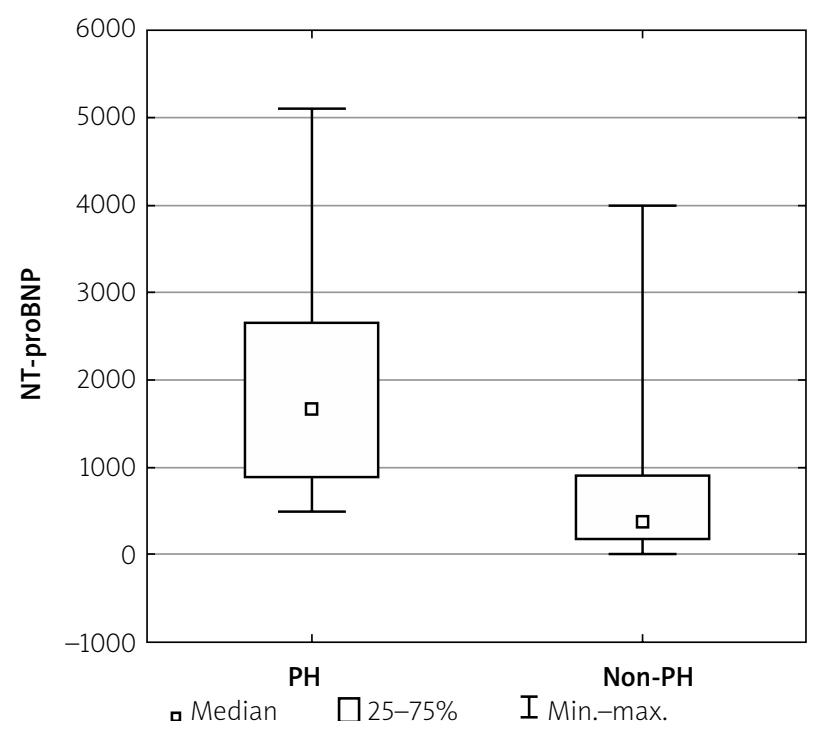

Figure 3. Patients with $\mathrm{PH}$ vs. non-PH had significantly higher levels of NT-proBNP (1664 (904.52679) vs. 367.3 (187.6-900), $p<0.000002)$

tween the NOHCM and PHOCM subgroups. We postulate that only stable long-term LVOT obstruction is sufficiently potent to highly elevate this biomarker, both when measured as the absolute value and when calculated by the standardisation value. In a recent study by Blackshear et al. [9] among highly selected patients (i.e. with HCM in New York Heart Association class I), almost all patients had elevated BNP levels. There were no clear differences in BNP value between the following subgroups: obstructive, provocable-obstructive, and non-obstructive HCM patients (although the latter subgroup included only 4 patients) [9]. Our study included more symptomatic patients, who were classified into the subgroups of NOHCM, PHOCM, HOCM, and comparisons of NT-proBNP and NT-proBNP/ULN values were performed. The NT-proBNP level differences between POHCM and HOCM might help plan non-pharmacological septal reduction therapy (alcohol septal ablation or myotomy/myectomy). NT-proBNP is an objective and precise parameter in comparison to the subjective NYHA class. Consequently, POHCM patients with mildly-moderately increased NT-proBNP might be candidates for enhanced pharmacotherapy and careful monitoring of biomarkers during follow-up. A further increase in the biomarker level might be a signal to switch from pharmacotherapy to non-pharmacological treatment methods [10, 11].

In another one-way invasive intervention [12], it was reported that myectomy significantly reduced NT-proBNP level in HOCM patients, in which a time-dependent manner of decrease indicated myocardial remodelling of the heart after myectomy.

To our knowledge, both NT-proBNP and NT-proBNP/ ULN have been not studied in the PHOCM subgroup (studied only in the NOHCM and HOCM subgroups).

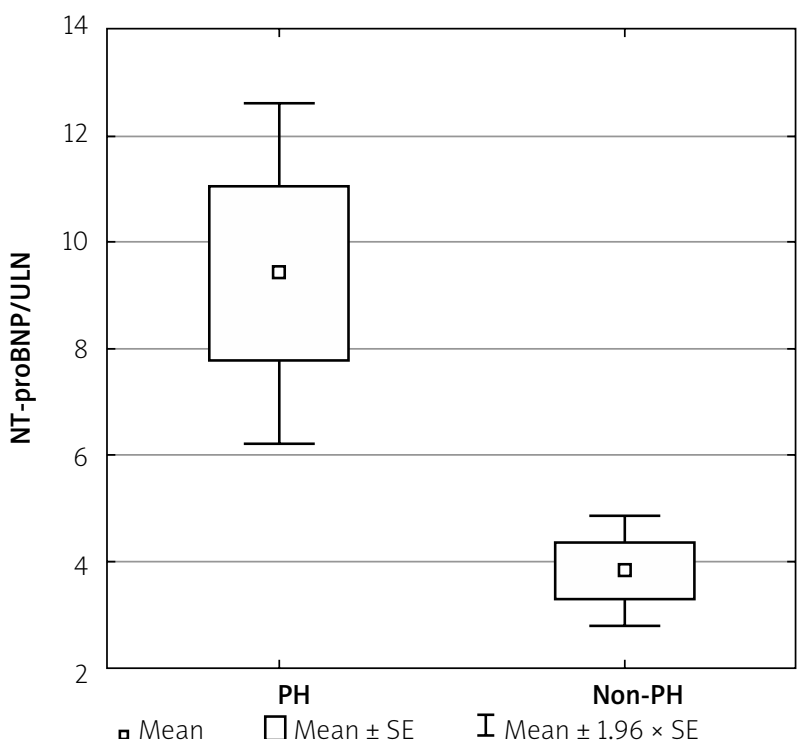

Figure 4. Patients with $\mathrm{PH}$ vs. non- $\mathrm{PH}$ had significantly higher levels of NT-proBNP/ULN (9.41 \pm 6.91 vs. $3.82, p=0.00011$ )

In a study by Coats et al. [13], the NT-proBNP concentration was not analysed in the PHOCM subgroup, and the authors [13] reported that NT-proBNP is a strong predictor of overall prognosis, particularly heart failure-related death and transplantation, but not of SCD. Similarly, a very recent, large study from China confirmed the relationship between elevated NT-proBNP levels and increased all-cause mortality or cardiac transplantation [14]. A subsequent study has reported that high NT-proBNP levels were not associated with an increased value of the calculated 5-year percentage risk of SCD in HCM (ESC calculator 2014) [15]. In contrast, an increased level of high-sensitivity troponin I, another popular cardiac biomarker, was shown to be associated with the risk of SCD in HCM via more frequent episodes of non-sustained ventricular tachycardia [15, 16].

In an Italian study [17] among a subgroup of stable outpatients with HCM, NT-proBNP proved a powerful independent predictor of death and heart failure-related events. Although the positive predictive accuracy of an increased NT-proBNP level was modest, low values reflected the true clinical stability, suggesting the possibility of avoiding or postponing aggressive treatment options.

The biomarkers used in HCM might reflect some haemodynamic pathologies from the right side of the heart. The presence of pulmonary hypertension is related to higher NT-proBNP values, but this biomarker is not related to RV size. It seems likely that a thickened and stiff interventricular septum restricts/compress the RV size/ geometry.

\section{Methodological consideration}

In the above-cited work by Coats et al. [13], as well as in a subsequent work by the same group [18], the 
authors take into account the influence of gender and age on NT-proBNP value. However, these authors did not divide the actual value by the ULN value to calculate the appropriate ratio of these values. Finally, Coats et al. performed a logarithmic transformation of their data. In 2018, Blackshear et al. [9] did calculate this ratio, and in this study we also use this method to obtain a normal statistical distribution. We propose that future studies should include this standardisation in their analyses.

\section{Limitations of the study}

There are two major limitations to this study: the small number of patients and the lack of prospective follow-up. Unfortunately, we do not have precise measurements of the right atrium. Additionally, we feel that the atrial natriuretic peptide (released only by atria) would have been a better biomarker for right atrium haemodynamic overload than the NT-proBNP (secreted by LV myocardium). Therefore, we propose that future work should assess the relationship between NT-proBNP and two parameters of right-side heart influenced by left ventricular and septal pathology.

\section{Conclusions}

Increases in NT-proBNP/ULN are related to larger LAD and LAA, while more elevated NT-proBNP is associated only with larger LAA. The highest levels of both NT-proBNP and NT-proBNP/ULN were associated with HOCM, whereas biomarker levels were comparably lower in PHOCM and NOHCM.

\section{Conflict of interest}

The authors declare no conflict of interest.

\section{References}

1. Elliott PM, Anastasakis A, Borger M, et al. 2014 ESC Guidelines on diagnosis and management of hypertrophic cardiomyopathy: the Task Force for the Diagnosis and Management of Hypertrophic Cardiomyopathy of the European Society of Cardiology (ESC). Eur Heart J 2014; 35: 2733-79.

2. Kehl D, Buttan A, Siegel RJ, et al. Clinical utility of natriuretic peptides and troponins in hypertrophic cardiomyopathy. Int J Cardiol 2016; 218: 252-8.

3. Costabel JP, Galve E, Terricabras M, et al. E/e' ratio and left atrial area are predictors of atrial fibrillation in patients with hypertrophic cardiomyopathy. Echocardiography 2018; 35: 935-40.

4. Gawor M, Śpiewak M, Kubik A, et al. Circulating biomarkers of hypertrophy and fibrosis in patients with hypertrophic cardiomyopathy assessed by cardiac magnetic resonance. Biomarkers 2018; 23: 676-82.

5. Zhang C, Liu R, Yuan J, et al. Predictive values of N-terminal proB-type natriuretic peptide and cardiac troponin I for myocardial fibrosis in hypertrophic obstructive cardiomyopathy. PLoS One 2016; 11: e0146572.

6. Dimitrow PP, Bober M, Michałowska J, et al. Left ventricular outflow tract gradient provoked by upright position or exercise in treated patients with hypertrophic cardiomyopathy without obstruction at rest. Echocardiography 2009; 26: 513-20.

7. Dimitrow PP, Rajtar-Salwa R. Obstructive form of hypertrophic cardiomyopathy-left ventricular outflow tract gradient: novel methods of provocation, monitoring of biomarkers, and recent advances in the treatment. Biomed Res Int 2016; 2016 : 1575130.

8. Galiè N, Humbert M, Vachiery JL; ESC Scientific Document Group. 2015 ESC/ERS Guidelines for the diagnosis and treatment of pulmonary hypertension: The Joint Task Force for the Diagnosis and Treatment of Pulmonary Hypertension of the European Society of Cardiology (ESC) and the European Respiratory Society (ERS): Endorsed by: Association for European Paediatric and Congenital Cardiology (AEPC), International Society for Heart and Lung Transplantation (ISHLT). Eur Heart J 2016; 37: 67-119.

9. Blackshear JL, Safford RE, Thomas CS, et al. Platelet function $m$ analyzer 100 and brain natriuretic peptide as biomarkers in obstructive hypertrophic cardiomyopathy. Am J Cardiol 2018; 121: 768-74.

10. Veselka J, Lawrenz T, Stellbrink C, et al. Low incidence of procedure-related major adverse cardiac events after alcohol septal ablation for symptomatic hypertrophic obstructive cardiomyopathy. Can J Cardiol 2013; 29: 1415-21.

11. Wojtarowicz A, Kornacewicz-Jach Z. Alcohol septal ablation in hypertrophic cardiomyopathy utilizing a longitudinal 17-year study (mean 10.8). Observation follow-ups taken at a single medical centre. Cardiol J 2017; 24: 125-34.

12. Cui H, Wu X, Wang S, et al. Time and age dependent decrease of NT-proBNP after septal myectomy in hypertrophic obstructive cardiomyopathy. Clin Chem Lab Med 2017; 55: 1614-20.

13. Coats CJ, Gallagher MJ, Foley M, et al., Relation between serum $\mathrm{N}$-terminal pro-brain natriuretic peptide and prognosis in patients with hypertrophic cardiomyopathy. Eur Heart J 2013; 34: 2529-37.

14. Meng XB, Wang WY, et al. Value of N-terminal pro-B-type natriuretic peptide on long-term outcome of patients with hypertrophic cardiomyopathy. Zhonghua Xin Xue Guan Bing Za Zhi 2018; 46: 192-7.

15. Rajtar-Salwa R, Hładij R, Dimitrow PP. Elevated level of troponin but not $\mathrm{N}$-terminal probrain natriuretic peptide is associated with increased risk of sudden cardiac death in hypertrophic cardiomyopathy calculated according to the ESC Guidelines 2014. Dis Markers 2017; 2017: 9417908.

16. Hładij R, Rajtar-Salwa R, Dimitrow PP. Associaton of elevated troponin levels with increased heart rate and higher frequency of nonsustained ventricular tachycardia in hypertrophic cardiomyopathy. Pol Arch Intern Med 2017; 126: 445-7.

17. D’Amato R, Tomberli B, Castelli G, et al. Prognostic value of $\mathrm{N}$-terminal pro-brain natriuretic peptide in outpatients with hypertrophic cardiomyopathy. Am J Cardiol 2013; 112: 1190-6.

18. Coats CJ, Parisi V, Ramos M, et al. Role of serum N-terminal probrain natriuretic peptide measurement in diagnosis of cardiac involvement in patients with Anderson-Fabry disease. Am J Cardiol 2013; 111: 111-7. 